\title{
Determining Significant Factors Affecting Reader's Perception of Writing Technique and Style: Views of Professional Writing Evaluators
}

\author{
John E. Knight \\ The University of Tennessee at Martin \\ Lajuan Davis \\ The University of Tennessee at Martin \\ Cooper Johnson \\ The University of Tennessee at Martin
}

\begin{abstract}
The evaluation of various writing styles continues to be of paramount importance in multiple venues. This research identified the most significant factors that affect the reader's perception of writing style in a business letter utilizing factorial statistical design of experiments (DOE). Based on these factors, several areas of teaching emphasis emerge as the most important issues in teaching and alternately in submitting any business letter or report. Using reader imaging, additional factors can be equally considered in the analysis of the quality of the business students' work. Extensions and limitations of the research are also discussed.
\end{abstract}

Keywords: Writing Style, Reader Perception, Business Letter

\section{INTRODUCTION}

The evaluation of various writing styles continues to be of paramount importance in multiple venues. Faculty judge student performance via submitted papers, businesses hire employees based on résumés and other written submissions, and various editors determine which papers will be published via the writtenword submissions. Many potential problems occur in the evaluation process. An individual evaluator can assign different grades (or perceptions) of a written passage if the writing is evaluated at different times (Knight, Tracy, \& Allen, 2010; Knight \& Allen, 2012). Further, different evaluators will judge a given paper to be written better or worse based on its content, technical presentation, and the writing style that appeals to them. The spectrum of evaluation can range broadly, based on individual reader perceptions, experiences, and feelings. However, the evaluation can also be based on statistical methods in addition to opinion. Statistical methods attempt to focus on demonstrating that certain patterns in the information can be extracted and proven to be repeatable and significant. This paper will demonstrate a technique for determining the statistically repeatable patterns in writing technique and style that can illustrate the factors that can be verified to be statistically repeatable and significant. The identification of those 
important factors can then lead to formulation of improvement strategies that can be used to improve students' writing style, technique, and reader perception.

\section{PURPOSE OF THE STUDY}

This study will utilize fractional factorial statistical design of experiments (DOE) in order to differentiate statistically the importance in reader perception of seven different writing factors: spelling errors, subject-selection errors, verb-selection errors, incomplete sentences, poor sentence variety, poor or missing transitions, and vocabulary difficulty. The reader outcomes will focus on the perception of writing styles from academic evaluators. Seven different evaluators with business writing knowledge and experience will be utilized with the emphasis of finding the factors that significantly influenced their evaluations.

The introduction of DOE allows for analysis of numerous different factors at a time versus testing the significance of one factor at a time (for example, do papers with more passive writing style versus active writing style affect the reader perception)? Past methods of testing one factor at a time basically try to imply a cause and effect in a simple framework. However, when other multiple factors are present, will the same statistical significance be exhibited? DOE has been found to be far more robust at identifying significant factors in the light of numerous other factors that are present. Additionally, DOE's have a unique ability to identify significant factors in the light of significant noise and multiple conflicting factors.

Statistical design of experiments has been utilized in the past to identify and isolate statistically significant factors in a wide array of business applications. However, the authors have not been able to identify any such application to the area of business writing styles. The methodology requires the development of some relevant writing-style factors, the organization of those factors into a formula (different combinations of writing factors such as spelling errors, subject-verb errors, etc. in a business document) and then the evaluation of the entire business document that has been revised to contain different combinations of errors, by different evaluators. The data from those different evaluators is then used to statistically determine those factors that statistically impact the reader's perception of which errors or combinations of errors prohibit students from being successful writers.

\section{LITERATURE SURVEY}

The literature of importance for this paper can be categorized into two areas: (1) importance of different writing factors in affecting a reader's assessment of success, and (2) design of experiments and the specialized ability to differentiate, in a robust manner, the statistical importance of different factors.

\section{Importance of Different Writing Factors Impacting Reader's Assessment of Success}

A reader's assessment of a written work is impacted by different writing factors and ultimately gives way to the determination of success by the author of the written work. Knight (n.d.) postulates that reader imaging is a critical factor necessary for success in relaying the full context and image intended from the author to the reader. In other words, in order for a writer to be successful in communicating the point of the work, he must provide the reader with a clear visual of the narrative, thus encouraging and promoting understanding.

The composition of the written piece contains many important parts that collectively are critical to the overall success of the final product. One writing factor identified as vital to the reader's assessment of success is the proper use of Standard English grammar (Hoggatt \& Lentz, 2017). In addition to grammar, the factors of spelling, organization, word choice, punctuation, and sentence structure are emphasized as requirements of achieving success through the eyes of the reader (Connolly, Hoggatt, \& Honl, 2003 as cited in Hoggatt \& Lentz, 2017). Furthermore using, clear, simple language rather than unnecessarily complex language is championed by Oppenheimer (2006) as use of simple language, he argues, can make authors be viewed as more intelligent. 
By studying the writing of students, additional factors have been acknowledged as contributing to writers' success. Jani and Mellinger (2015) studied the factors that influenced social work students' writing skills and determined that five main themes are predominate: Students' level of effort, students' relationship with the course content, the educational support students receive, students' perception of their roles in the learning process, and students' perceived self-efficacy regarding their writing ability. These themes identify the need for students, as writers, to actively participate in writing, including a desire to be successful, be interested in the subject matter, seek and receive appropriate assistance with their writing efforts, understand their tasks as writers, and be confident in their skills as writers.

Moreover, writing is influenced by other factors that then impact the author's quality and complexity of writing, as observed by the readers of their work. According to Douglas and Miller (2016) a secondary factor to writing is the quality of the content authors read. Specifically, with regards to student writers, the more quality the material to which they expose themselves, the better quality their written output becomes. Douglas and Miller, more specifically, shared that student writers who immersed themselves in reading "academic journals, 'literary' fiction, or general non-fiction wrote with greater syntactic sophistication than students who read genre fiction (mysteries, fantasy, or science fiction) or exclusive reading of web-based contend from the likes of Reddit, Tumblr, and BuzzFeed" (p. 77).

\section{Design of Experiments and the Specialized Ability to Differentiate the Statistical Importance of Various Factors}

The statistical methodology selected for this research was a special category of fractional factorial designs called Plackett-Burman (PB) designs (Montgomery, 1991; Tyssedal, 2008; Wheeler, 1998). Plackett-Burman experiments are based on $2^{2}$ designs. These designs allow for the development of a range of experimental designs based on formula patterns of multiples of 4 items, 8 items, 12 items, and more. These designs have tremendous analytical power based on relatively few samples given the specialized design of the experiments. The reduction from full factorial designs to fractional factorial designs provides for the examination of main effects of factors while only gaining minimal insight into interactions in the factors. The loss of analytical power in interaction also allows for a much smaller sample size experiment. Most importantly, multifactor designs, like Plackett-Burman designs, test more than one factor at a time and in the presence of other potentially significant factors so that robustness of results is facilitated. Applications of these designs to business abound (Koselka, 1996; Pande, Neuman, \& Cavanagh, 2000; \& Knight, 2016).

\section{BASIC MODEL FORMULATION}

In order to test multiple factors at a time using the Plackett-Burman designs, a general model structure needs to be contemplated. Since PB designs evolve in steps of 4 , a design needed to be either 4, 8, 12 , etc. factors. The decision as to which model would be best hinges on several factors. In this case, multiple different factors of writing can be contemplated. However, at the same time, consideration must be given to the number of formulas (documents with different combinations of errors) that will be required to be developed and evaluated. The number of formulas to be developed will also be $4,8,12$, etc., while the associated number of potential factors that can be examined in these models is (n-1), factors based on the model size, e.g. 3, 7, 11, etc. Further, consideration must be given to the individuals who will grade the business documents and assign a numerical measure to each one. The decision was made in this case to select a size 8 PB design that will be capable of evaluating seven (7) grammatical factors. Thus, each evaluator will be given eight (8) different combinations of grammatical errors in the same business letter and will be asked to provide an overall grade for each letter based on their impression of the different combinations of errors. Once the grading is completed, statistical analysis of the results of multiple graders will provide the necessary conclusions on statistically significant factors that affect the reader's impression of the paper. Although other considerations such as mid-points and reflection can be considered, those approaches only add to the number of formulas (combinations of errors) that need to be developed and evaluated. Given the literary form of each formula (and not numerical results from an 
industrial experimentation), assigning a total of eight business documents containing a combination of different errors to each evaluator was deemed to be sufficient (repetitive enough) because finding readers to evaluate the papers would be difficult enough without adding to the total number of formulas.

The basic structure for an 8 factor PB (that can test as many as 7 unique factors) is provided in Table 1.

TABLE 1

PLACKETT-BURMAN 8 FACTOR DESIGN

Plackett-Burman 8 factor design

\begin{tabular}{|c|c|c|c|c|c|c|c|c|c|c|c|c|c|}
\hline Recipe & A & B & C & D & E & F & G & Y1 & Y2 & Y3 & Y4 & Y-BAR & R \\
\hline $\mathbf{1}$ & 1 & 1 & 1 & -1 & 1 & -1 & -1 & & & & & & \\
\hline $\mathbf{2}$ & -1 & 1 & 1 & 1 & -1 & 1 & -1 & & & & & & \\
\hline $\mathbf{3}$ & -1 & -1 & 1 & 1 & 1 & -1 & 1 & & & & & & \\
\hline $\mathbf{4}$ & 1 & -1 & -1 & 1 & 1 & 1 & -1 & & & & & & \\
\hline $\mathbf{5}$ & -1 & 1 & -1 & -1 & 1 & 1 & 1 & & & & & & \\
\hline $\mathbf{6}$ & 1 & -1 & 1 & -1 & -1 & 1 & 1 & & & & & & \\
\hline $\mathbf{7}$ & 1 & 1 & -1 & 1 & -1 & -1 & 1 & & & & & & \\
\hline $\mathbf{8}$ & -1 & -1 & -1 & -1 & -1 & -1 & -1 & & & & & & \\
\hline
\end{tabular}

In Table 1, the different factors to be tested are labeled A, B . . G while the different formulas are labeled 1 through 8 . Each writing style evaluator will be given 8 versions of a business letter to read and grade. The eight grading results for the business letter with eight different versions of errors will be inserted under Y1 for the first grader, under Y2 for the second grader and so forth. Based on the total number of graders, each business letter will develop an average value from the scores of the different graders (called Y-Bar) along with a measure of variation for each combination of errors (call R). The actual statistical analysis will then evolve based on those basic data values for mean and dispersion.

The values of one (1) and minus one (-1) in each formula reflect the inclusion or deletion of a chosen factor in each formula or each version of the business letter. The development of different combinations of errors in the business letter will be made clearer as the factor table with the various intensity levels is presented.

\section{FACTOR TABLE AND INTENSITY LEVELS}

For this application of $\mathrm{PB}$, seven different factors examined based on the literature survey of writing factors that were judged to be critical in creating writing style and technical competence.

Table 2 lists the factors that were selected and the intensity levels that were chosen as the factors to be tested. 
TABLE 2

FACTORS TO BE TESTED

\begin{tabular}{r|ll}
\hline \multicolumn{1}{l}{ LABEL OF } & \multicolumn{1}{l}{ TYPE OF ERROR } & NUMBER OF ERRORS \\
FACTORS & & 5 \\
\hline A & Spelling errors & 3 \\
B & Subject selection errors & 4 \\
C & Verb selection errors & 2 \\
D & Incomplete sentences & 2 \\
E & Poor sentence variety & 2 \\
F & Poor or missing transitions & 5 \\
G & Vocabulary errors & \\
\hline
\end{tabular}

In Table 2, factor A serves as an example to the methodology of formula building in the design of experiments. Spelling errors are a matter of little dispute and so the discussion is straightforward for using this element. As for intensity levels, in exactly four of the eight formulas, no spelling errors exist. In the remaining four formulas, exactly five misspellings were included. Now, referring to the Plackett-Burman 8 factor design of experiments table, each evaluator of the various business letters will be exposed to either spelling errors or no spelling errors. In formula \#1, the plus one means that business letter one will have five spelling errors spread throughout the business letter. (The actual writing style of the grading exercise focuses on a business letter that will be discussed later and included in Appendix). In formulas (business letters) two and three, the minus one (-1) means that the letter has zero misspellings. This assignment pattern continues to be applied to the remaining formulas with the plus one (1), meaning that the intensity factor has the stated number of errors while the minus one (-1) indicates that the factor has zero errors in the business letter of that particular type, although as will be discussed further, other factor errors will be present as stipulated by the plusses and minuses in the experimental design.

Factor B in the study consists of subject selection errors while Factor C is verb selection errors. Subjects and verbs are the building blocks of writing with message clarity depending on the choice of both elements. "Nothing reveals a person's education, or lack thereof, so quickly as verbs that don't agree with subjects," (Guffey \& Seefer, 2014, p. 136). In business writing, students are taught to write documents in a brief, clear, and concise manner. The clearest messages contain proper, identifiable subjects, followed by verbs that agree with the subjects in number and person. Subjects must be easy to locate (identify), recognized by number (singular or plural), and agree in number with the verb, which should also be easy to locate or identify.

Factor D consists of incomplete sentences. For a sentence to be correct and a message to be clear, the sentence must have a subject, a verb, and must make sense. Too often writers do not check their sentence structure for the standard subject-verb order or agreement resulting in incomplete sentences or thoughts. Incomplete sentences can be categorized as phrases or clauses, but not as sentences until they meet the criteria of having a subject, a verb, and making sense.

Factor E is poor sentence variety. In order to make messages more interesting and "readable," writers should use a variety of sentence structures. Writing a document in short, simple sentences can make the document seem choppy; however, combining dependent clauses with independent clauses or combining two independent clauses, etc. can add a variety to the structure of sentences that provides more pleasurable, but still accurate, reading.

Factor F in the study addresses poor or missing transitions. Coherence in writing can be achieved by "(a) repetition of key ideas or key words, (b) the use of pronouns that refer clearly to their antecedents, and (c) the use of transitional expressions" (Guffey \& Seefer, 2014, p. 161). The transitional expressions serve as road signs to dictate the direction of the message and to move the reader in a cohesive direction. Examples of some transitional expressions are the words or phrases although, consequently, however, of course, for example, etc. 
Finally, Factor $\mathrm{G}$ is vocabulary errors. Having an appropriate vocabulary is an asset in business writing. Individuals who understand the meaning of many words and how to use them, have an advantage over readers and writers who are not as prolific. Knowing the correct words to use in a message can assist the writing in producing messages that are brief, clear, and concise (Guffey \& Seefer, 2014).

Now, referring to the Plackett-Burman 8 Factor Design Table 1, the eight different formulas that are being tested can be viewed in totality. As an example, formula number one will be a business letter based on the letter shown in Appendix with the following combination of errors: five spelling errors (note the plus one under factor A); three subject-selection errors; four verb-selection errors; no incomplete sentences (note the -1 under Factor D); two sentences with poor variety; no poor or missing transition; and no vocabulary errors.

Formulas two through eight incorporate the seven factors $\mathrm{A}$ through $\mathrm{F}$ according to the pattern represented for that respective formula with the inclusion of the factor at the intensity level shown or zero of the factor in the formula. The result is a statistically designed experiment in which the different factors and their intensity levels occur in a variety of ways so that the main effects of those factors on a reader's perception of the business letter can be determined. Note that the errors occur in the presence of other factors and potential presence or non-presence. Thus, noise is incorporated into each formula (and thus in the reader's mind), and the relative weights of different writing errors are being judged in the totality of the mix of other factors.

\section{RESEARCH MEDIUM AND RELATED STATISTICS}

The business correspondence letter (see Appendix) served as the basis for the designed experiment. In the study, this letter represents formula eight (8), a formula in which no deficiencies exist in any category. For example, the letter contains zero spelling errors, zero subject selection errors, zero verb selection errors, zero incomplete sentences, etc. All of the other formulas (formulas 1 - 7) have various combinations of the errors. An analysis of the letter for related statistics for the letter appears in Figure 1.

\section{FIGURE 1 \\ READABILITY STATISTICS OF BUSINESS LETTER}

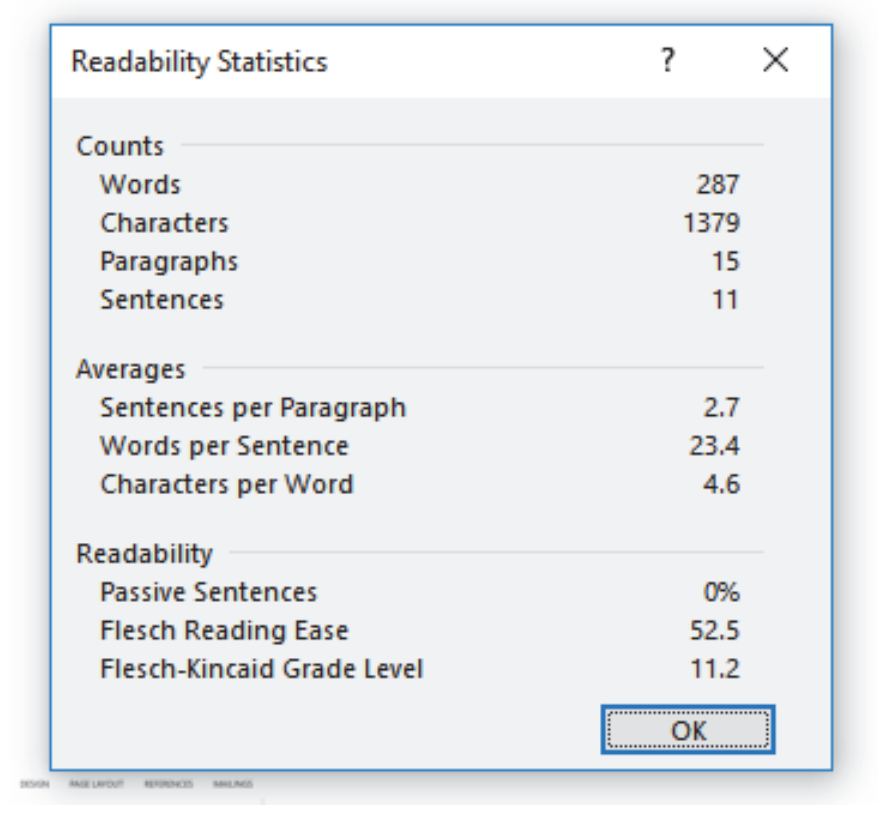


For this letter, the statistics indicate a reading difficulty on the 11.2 grade level. This level designation seems acceptable for college-level students to read and for academic evaluators to rate.

\section{ADMINISTRATION OF THE EXPERIMENT}

Selection of evaluators as well as assessment methodology is explained in the following sections. Nine university professors participated in this study, were given detailed instructions on their assessment responsibilities, and the results of their work were then thoroughly analyzed.

\section{Randomization of Formulas}

Given the eight created formulas, many additional factors other than the seven factors present may potentially affect the readers' assessment of the business letters. The most effective way to minimize the effect of these other factors dictates that the formulas be given to the reading evaluators in a random order. The randomization of the eight formulas essentially averages out other effects that may be present. In this case, each formula was assigned a different color and the packet of formulas was provided to each reader in a randomized order.

\section{Selection and Educational Distribution of Reading Assessment Evaluators}

Reading evaluators included nine college professors. Four of the evaluators participated actively in teaching and evaluating business report writing. The remaining five evaluators taught other courses in business fields that required writing assessments. Faculty from The University of Tennessee at Martin and Columbia College of Chicago, Illinois, graded the eight different formulas from the design of experiments.

\section{Instructions to Evaluators and Assessment Methodology}

Each evaluator was provided with a packet of the eight different formulas. The formulas were randomly mixed in the packet and a grading sheet for the different colors was provided. The evaluators were instructed to read the letter and provide a grade from 55 to 100 based on A+ to F. Each grade was subdivided into three parts, for example, A+, A, and A-. The numerical values for each letter grade assessment were then converted to a numerical value, e.g. A+ equals 97.5, A equals 95 and A- represents 91.5. The other grade levels (B through F) were evaluated similarly.

Each instructor was asked to read each paper without any editorial markings or judgements and then asked to provide a grade. In order to prevent readers from having different physical conditions and prejudices, each evaluator was asked to allot adequate time to read and grade all of the papers at one sitting. The results for all nine of the graders were then entered into a 7 factor Plackett-Burman calculation routine to provide the basis for analytical results.

\section{ANALYSIS OF THE EXPERIMENT'S MAIN EFFECTS}

The raw data from the experiment are summarized in Table 3. The values for each value of Y (Y1 to Y9) represent the individual grades that an evaluator assigned to each formula. Although these scores each appear to be in sequence for each formula, the grades were actually assigned to different color assignments and compiled in random order. Thus, formula 8 (which had theoretically no deficiencies in the seven factors) was not the last formula read by each evaluator. However, note the grades for formula 8 appear to be consistently high relative to the other formulas graded by the evaluators. In other words, each evaluator read without comment or editing each of the formulas in random order but still seemed to choose the best formula and assign a rather consistently high grade. In fact, the average for formula 8 for all nine evaluators was 92.55 (find the cell intersection of formula 8 and Y-Bar), which was thus judged to be the best letter in the batch of eight random letters. 
TABLE 3

SUMMARY OF RAW DATA FROM THE EVALUATORS

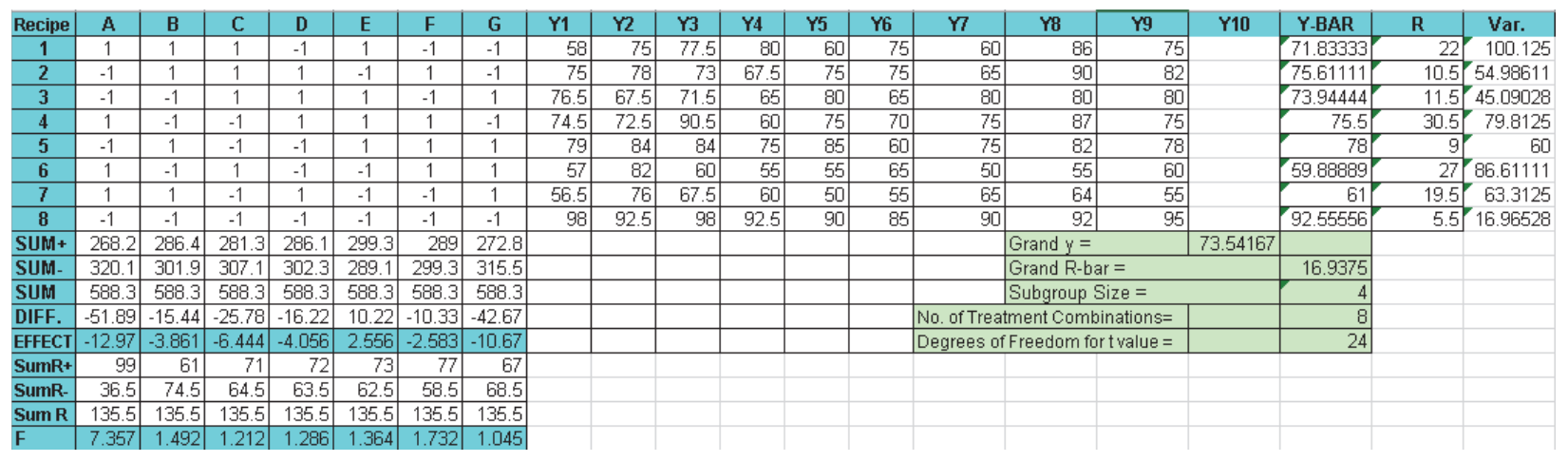

The goal of the analysis is to determine those factors that significantly reduce the reader's evaluation of a paper and to what extent each factor plays in diminishing the reader's assessment of the final business letter. The "reduction" is based on the fact that formula 8 (with all minuses in the factor columns) indicates that formula 8 has no errors and thus the presence of any other factor will reduce the grade for that paper (or at least expectedly so and in this case did just that). The appeal of the experimental design hinges on the fact that each paper confounds different deficiencies in each formula and the reader must judge the letter not just based on a personal predisposition to a particular deficiency, but a deficiency confounded with other factors.

The initial step of the analysis focuses on identifying the random variation within the experimental design. The identification of significant factors depends on those factors overcoming the random variation that is always present in any evaluation, most especially evaluations relating to qualitative assessments (Knight \& Allen, 2012). The random variation within the experiment is determined as follows:

$\sigma_{\exp }=\frac{\bar{R}}{d_{2}}=\frac{16.9375}{2.06}=8.222$

The value of $\mathrm{d}_{2}$ is a constant from statistical tables related to the sample size. In this case, the sample size of four represents the number of values composing the plus and minus averages in each factor.

The next step focuses in determining the standard deviation of the effect (or standard deviation of the sample means of the different factors). The following formula applies to the data collected:

$\hat{\sigma}_{\text {effect }}=\hat{\sigma}_{\text {experiment }} \sqrt{\frac{4}{N}}=8.222 \sqrt{\frac{4}{8 * 9}}=1.937$

Note: $\mathrm{N}$ equals the total number of data points that includes 8 formulas and 9 observations on each formula.

Control limits on the effects result from developing confidence intervals based on the standard deviation of the effect. The following formula provides the values for the control limits:

ControlLim itsfortheEffects $= \pm t \hat{\sigma}_{\text {Effect }}= \pm 1.998 * 1.937= \pm 3.87$

The value of $t$ is based on a 95\% confidence where $t$ is based on a degrees of freedom of ( $n$ $1) *($ number of treatment combinations $)$ or $\mathrm{d} . \mathrm{f} .=(9-1)(8)=64$. 
Another important consideration results from calculating the average score provided by the readers at each level of factor inclusion ( -1 means not included and +1 means included). The averages are calculated by locating the SUM+ values under Factor A (for example) and dividing by four (4) since four formulas existed with the +1 value in column A. The same procedure would be used to determine the average for the -1 values. The values for each factor are summarized in Table 4. The EFFECT is the difference between the average reader score for the letter with no defects ( -1 formulation) and the average reader score for the letter with the formulated number of defects of factor A to $\mathrm{G}$.

TABLE 4

SCORE VALUES OF FACTORS A - G AT PLUS AND MINUS LEVELS

\begin{tabular}{l|llllllll}
\hline FACTOR & \multicolumn{1}{c}{ A } & \multicolumn{1}{c}{ B } & \multicolumn{1}{c}{ C } & \multicolumn{1}{c}{ D } & E & \multicolumn{1}{c}{ F } & G & \\
\hline (-) AVG. & 80.03 & 75.47 & 76.76 & 75.57 & 72.26 & 74.83 & 78.88 & -1 \\
(+) AVG. & 67.06 & 71.61 & 70.32 & 71.51 & 74.82 & 72.25 & 68.21 & +1 \\
EFFECT & -12.97 & -3.8661 & -6.444 & -4.056 & 2.556 & -2.583 & -10.67 & \\
\hline
\end{tabular}

As an example, if one considers Factor A: When that factor was included correctly (no defects), then the average score was 80.03. When Factor A was included in the letter at the required intensity, the average score of the four evaluations was 67.06. Thus, the differential (EFFECT) of having Factor A included was a reduction of 12.97 in the grade.

Combining the information on control limits for the effects and the actual effects can be used to generate a visual analysis of the factors that are statistically significant from those that are not statistically significant at the 95\% confidence level $($ alpha $=.05)$ (see Figure 2).

FIGURE 2

\section{CONTROL CHART OF EFFECTS}

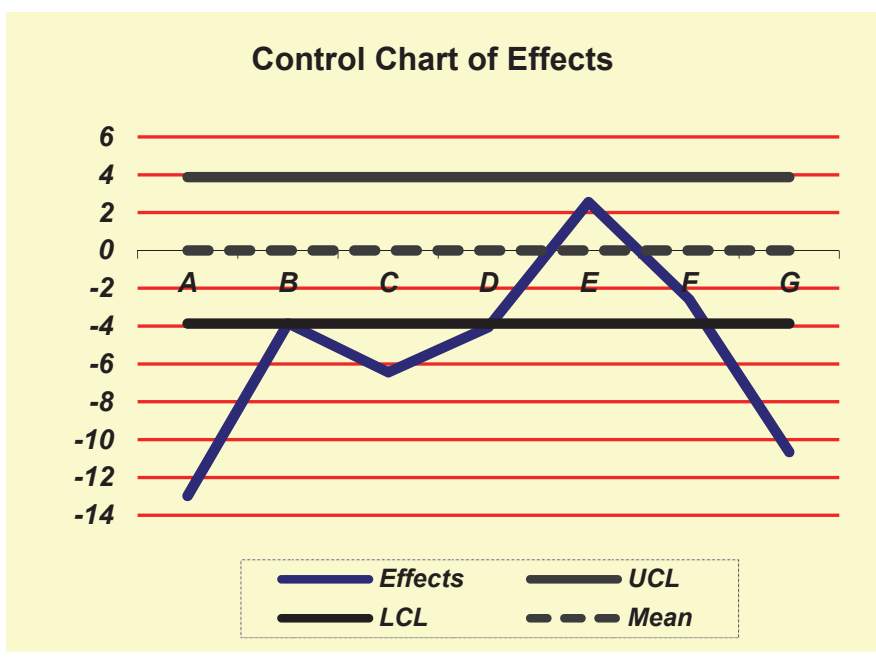

The interpretation of the chart rests on the fact that those factors that fall within the control charts for effects are not statistically significant at this point, while those outside of the control limits are statistically significant at the 5\% level or less. Thus, visually Factor A is extremely statistically significant at reducing the average grade that will be assigned by the evaluator when reading and mentally evaluating the business letter.

A Pareto chart of the different effects emphasizes the relative importance of focusing on eliminating a given writing deficiency in the mind of an evaluator. Thus, the presence of factors A, G, and C (and 
possibly D and B to a lesser extent) remain the primary factors in diminishing a reader's evaluation of the writing style in the letter (see the Figure 3).

\section{FIGURE 3 \\ PARETO CHART OF EFFECTS}

Pareto Chart of Effects

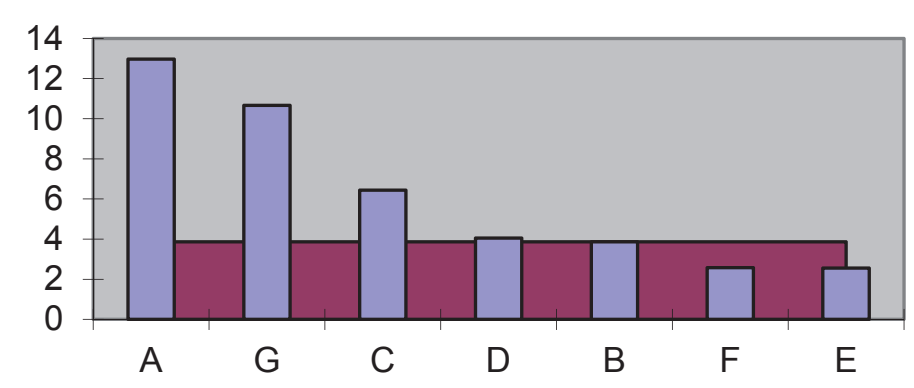

Referencing the table of Errors (and Intensity Chart), Table 2, spelling errors most significantly diminishes a reader's perceptions while vocabulary errors is the second most significant factor and verb selection errors also being a significant factor in reducing the reader's perception of the writing technique and style.

Next, the linear regression graph of each of the statistically significant effects can be plotted with the assumption that the effect of more of the factor is linear. For example, a graph of the average assessment reduction versus the number of spelling errors is shown. Given that the effect from $(-1)$ to $(+1)$ is -12.97 , then the reduction per individual spelling error (the slope of the line) would be -2.60 per spelling error (see Figure 4).

FIGURE 4

LINEAR REGRESSION OF STATISTICALLY SIGNIFICANT EFFECTS

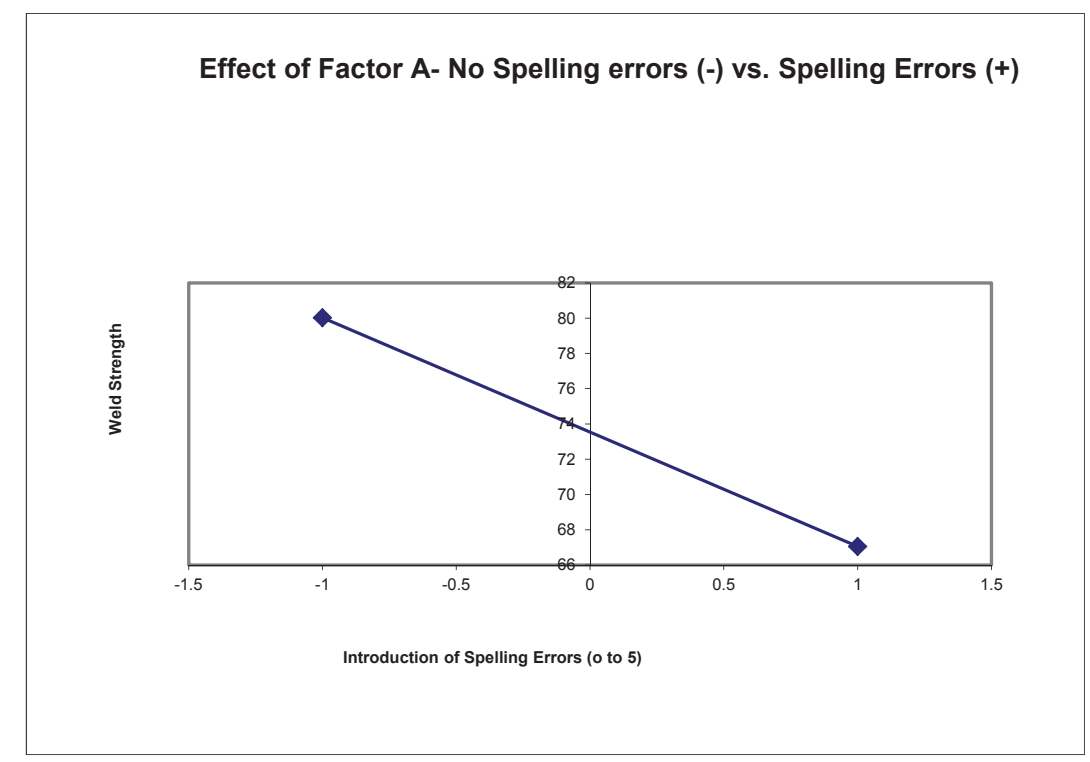


The graph in Figure 4 is designed to assume that a "linear effect" exists in the grade reduction. Although the assumption was not tested with midpoints in the experimental design, the assumption is generally reasonably realistic in DOE experimentation.

A similarly negatively sloping graph for Factor $G$ (vocabulary errors) yields a total negative effect for the factor of -10.67 . Again, given that the effect from -1 to $(+1)$ was -10.67 , then the rate of decrease in reader evaluation was -2.13 per vocabulary error.

The same type of graph would occur for Factor $\mathrm{C}$ (verb selection errors). The total effect for the factor was -6.44 from 0 (zero) verb selection errors to three verb selection errors. Thus, the rate of decrease assuming linear effects would be -2.15 per verb selection error.

At this point and given the sample size of nine evaluators, the reoccurring factors are either not significant at the 5\% level or barely significant. Thus, at this point, these factors fall within the random variation of the experiment and conclusions, as to their effect, are not statistically significant. Interestingly, Factor E indicates a "positive sloping effect," thus indicating that "poor sentence variety" might be preferred. However, the experiment clearly shows that the effect is in the realm of random variation at this point and is subject to no statistical conclusion as to the effect. In fact, larger sample sizes may indicate the same or different result, thus the result is indeed random.

The remaining factors $\mathrm{D}, \mathrm{B}$ and $\mathrm{F}$ (subject selection, incomplete sentences, and poor or missing transitions respectively) do not, at this point, show themselves to be statistically significant. However, the result is (as are all statistical conclusions) sample-size dependent. Given large enough sample sizes, even the most minor average differences can be shown to be statistically significant although the results may not prove to be practically significant in terms of plans for action based on the experiment. Given the relatively small sample size in this confounded experiment, the significant factors have emerged very quickly and, thus, are quite important.

Although interaction of factors can be suggested (but not statistically proven) by fractional factorial designs, the interpretation of any such interactions given the relative interpretive factors in writing style would be extremely difficult to interpret and use. Thus, for this paper's purpose, the investigation of interaction was omitted. However, if past DOE's indicate trends, most interactions would most likely occur between the statistically significant factors.

\section{A PROGRAM FOR REALLOCATING TEACHING EMPHASIS BASED ON RESULTS}

Based on the most significant factors that affect the perception of writing style in a business letter, several areas of teaching emphasis emerge as the most important issues in teaching and alternately in submitting any business letter or report.

First, the most significant factor was spelling errors. Obviously, students need at all times to be aware that the submission of any paper needs to be spell checked and grammar checked for errors (and conversely, correctness). Most standard word processing programs have spell checkers. The importance of actually using these tools must be reinforced and emphasized. Thus, the first factor of spelling correctness has a relatively easy "fix" as educators strive to place continual emphasis on using the actual analytical tools that are available.

Second, vocabulary errors were significant. Knowing and using proper vocabulary can be accomplished via technology as well. While grammar checkers on word-processing programs will flag some errors, students can also use synonym checkers to verify word choices. Electronic synonym checkers can be downloaded to cellular phones for use in proofreading messages for vocabulary errors. Additionally, all students should be encouraged to read books on a more consistent basis. Reading a variety of books, newspapers, trade journals, etc. can assist students in improving and broadening their vocabulary.

Lastly, verb selection errors were significant in the study as well. As previously discussed, messages cannot be clearly written or understood if appropriate verb selection is not accomplished. Students/writers can simply ask, "What do I want/need the subject to "do"? Answering the question by selecting an action verb from a printed list of verbs can help improve students' verb choices in writing. Of course, students 
must ensure that the verb selected agrees with the number and person of the subject. A proficient grammar checker in a word-processing program can assist the writer with verb selection decisions.

\section{EXTENSIONS AND LIMITATIONS OF THE RESEARCH}

The model provides a grading estimate based on the significant factors. Based on the theory developed from multiple regression analysis as applied to fractional factorial design, the following predictive equation would result from the analysis.

$\hat{y}=\bar{y}+\frac{1}{2} E(A) A+\frac{1}{2} E(G) G+\frac{1}{2} E(C) C+\varepsilon$

where $\hat{y}=$ the estimated average rating score of the writing letter

$\bar{y}=$ the average estimate of the evaluation score,

$\mathrm{E}(i)=$ the average effect of factor $i$.

$\mathrm{A}=$ the effect $(+1)$ or $(-1)$ based on the presence or non-presence of the factor.

Note that in this case, A could be a ratio of +1 or -1 since the effect may not be present in totality (e.g., two spelling mistakes instead of the factor inclusion in intensity of five mistakes). Thus, a partial ratio of the effect could be present.

Based on this formulation, the equation can predict the best estimate of reader perception based on the identification of the number of misspellings, the quantification of poor vocabulary instances, and finally the number of poor verb selections.

For example, suppose that the paper contains no perceived errors. Then, the estimated average score that would be given by evaluators can be calculated as follows:

$\hat{y}=73.54+\frac{1}{2}(-12.97)(-1)+\frac{1}{2}(-10.67)(-1)+\frac{1}{2}(-6.44)(-1)=88.57$

As another example, if the paper contained two misspellings, three poor examples of vocabulary and three poor verb selections, then the final predictive average value of the paper would be as follows:

$\hat{y}=73.54+\frac{1}{2}(-12.97)(+1)\left(\frac{2}{5}\right)+\frac{1}{2}(+10.67)(+1)\left(\frac{3}{5}\right)+\frac{1}{2}(+6.44)(+1)\left(\frac{3}{4}\right)=65.33$

Obviously, some variation in the identification or interpretation of poor vocabulary and poor verb selection can cause some discrepancy in the best guess estimate.

Further, an analysis of the variance of each factor can add greater understanding of the factors affecting the perception of the overall evaluation of the business letter. F values for each factor are calculated as follows:

$F=\left(\frac{\sum R_{+}}{\sum R_{-}}\right)^{2}$ or $F=\left(\frac{\sum R_{-}}{\sum R_{+}}\right)^{2}$ whichever is $\geq 1$ 
These values were calculated at the bottom of the basic tabulation table given earlier in the paper. These values then need to be compared to $F_{\text {crit }}$ for the number of degrees of freedom given by the following formula:

df. $=(n-1)$ Pumber of Treatment Combinations

In this case, nine evaluators (n) were used and eight treatment combinations were formulated. Thus, the total degrees of freedom would be d. f. $=(9-1)(8 / 2)=32$ for both the numerator and denominator. The value for $\mathrm{F}$ critical for $5 \%$ and 30 degrees of freedom would be as follows.

$\mathrm{F}_{\text {crit }, 30,30,05}=1.84$

A pareto distribution of the $\mathrm{F}$ values for each factor versus the critical value is shown in Figure 5 .

\section{FIGURE 5 \\ PARETO CHART OF VARIANCE EFFECTS}

Pareto Chart of Variance Effects

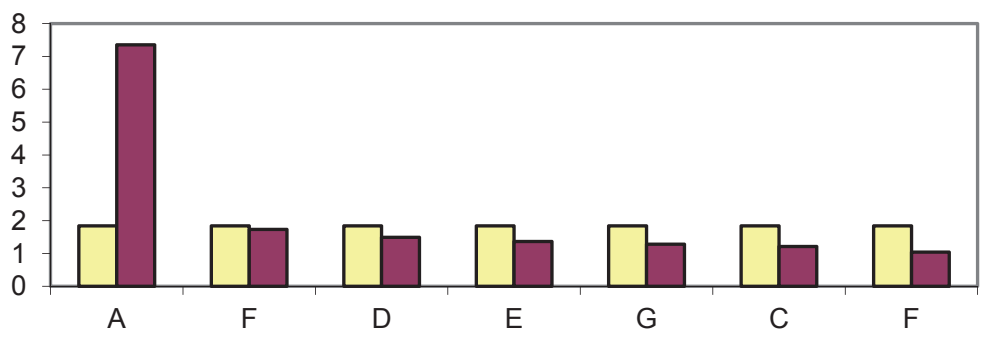

Figure 5 indicates that Factor A has significant variance differences in the grade evaluations from having spelling errors present to not having spelling errors present. In fact, when spelling errors are present at the indicated intensity level, not only is the average score on the evaluation lower but the variation in assigning grades is significantly higher. Thus, the presence of spelling errors seems to greatly impact certain of the evaluators more than other of the evaluators. The significance of the observation means that the enforcement of spelling checkers and elimination of spelling errors not only will increase grade evaluations but also lower the amount of variation in the overall grade assignments. None of the other factors proved to be significant in variance differences at the $5 \%$ level.

Finally, the data collected in the experiment leads to an estimate of the standard deviation of the estimated values from the regression equation and thus a confidence interval on the potential assigned values for any best guess of the grade given the presence of the significant factors. The formula for the evaluation is shown below along with the associated values. The value for $d_{2}$ can be found in standard statistical quality control tables based on different sample sizes (in this case, $n=9$ based on the nine evaluators).

$\sigma_{\text {experiment }}=\frac{\bar{R}}{d_{2}}=\frac{16.93}{2.97}=5.70$

Given this variation, a 95\% confidence of any evaluation of a paper is subject to a precision estimate as follows: 
precision $= \pm t_{n-1} \sigma_{\text {experiment }}=( \pm 1.86)(5.70)= \pm 10.60$

Thus, any paper would have a range of plus or minus around 10.60 points given a best guess estimator of the paper based on its characteristics. For example, the paper mentioned with two spelling errors, three vocabulary errors, and three verb selection errors would range between $65.33 \pm 10.60$ points in the final grading by any individual evaluator with $95 \%$ confidence.

Although the research is still in the developmental stage, the usefulness of a fully developed model would provide an assessment estimate from a student submitting a paper to an algorithm that can detect misspellings, poor vocabulary, and poor verb selection. Since artificial intelligence algorithms are being developed today at a rapid pace, the model research would serve as a step in a system to pre-emptively assess a paper and offer suggestions for improvement.

Further models using the same methodology can be envisioned. Data for student responses to the business report-writing example have been gathered which will allow further analysis as to student responses to their evaluation perceptions of the same letter. While those should provide insight into the thought process of students, the data will also allow the comparison of that data with the academic evaluators. Differences between the students and faculty evaluations can then be addressed. Further, the most important time period in a student's life occurs after graduation and in the actual work environment. Having the same set of papers evaluated by working business men and women will provide a further comparison that will help validate the model to the employers of the students that academicians produce and teach.

\section{CONCLUSION}

Through utilizing reader imaging in grading, far more factors can be equally considered in analysis of the quality of the business students' work. The application of the algorithms to detect specific common issues would allow for a quicker turnaround time between student submissions and faculty feedback and grading. Additionally, the appreciation of the identification of the grammar and spelling errors would be transferable to areas outside of business, such as English, communications, sciences, and social disciplines. While these results show promise, additional research is needed to confirm the results on a larger scale. 


\section{REFERENCES}

Douglas, Y., \& Miller, S. (2016). Syntactic Complexity of Reading Content Directly Impacts Complexity. International Journal of Business Administration, 7(3), 71 - 80. Retrieved May 31, 2018, from http://www.sciedupress.com/journal/index.php/ijba/article/view/9481/5736

Guffey, M. E., \& Seefer, C. (2014). Business English, 11e. South-Western Cengage Learning. Mason, $\mathrm{OH}$.

Hoggatt, J., \& Lentz, P. (2017, April). Who's responsible for teaching grammar, mechanics, and punctuation to business students? Business Education Forum, pp. $14-17$.

Jani, J. S., \& Mellinger, M. S. (2015). Beyond "Writing to learn": Factors influencing students' writing outcomes. Journal of Social Work Education, 51(1), 136 - 152. doi:10.1080/10437797.2015.977177

Knight, J. E. (2016). Improving quality using Plackett-Burman Screening Designs. The Business Review, 24(2), $8-14$.

Knight, J. E. (n.d.). Improving student business report writing through reader imaging: Guidelines and results of application. Unpublished manuscript, Department of Management, Marketing, and Information Systems, University of Tennessee at Martin, Martin, TN.

Knight, J. E., \& Allen, S. (2012). Establishing consistency measurements of grading for multiple section courses. Journal of the Academy of Business Education, 13, 28 - 47.

Knight, J. E., Tracy, D., \& Allen, S. (2010). Using Six Sigma methods to evaluate the reliability of a teaching assessment rubric. The Business Review, 15(1), $1-6$.

Koselka, R. (1996, March). The new mantra: MVT. Forbes Magazine.

Montgomery, D. C. (1991). Design and analysis of experiments. John Wiley and Sons, NY.

Oppenheimer, D. M. (2006, March). Consequences of erudite vernacular utilized irrespective of necessity: Problems with using long words needlessly. Applied Cognitive Psychology, 20(2), 139 - 156. doi:https://doi.org/10.1002/acp.1178

Pande, P., Neuman, R., \& Cavanagh, R. (2000). The Six Sigma way: How GE, Motorola and other top companies are honing their performance. India: McGraw Hill.

Tyssedal, J. (2008). Plackett-Burman Designs. In F. Ruggeri, R. S. Kenett, \& F. W. Faltin (eds.), Encyclopedia of statistics in quality and reliability. United States: John Wiley and Sons.

Wheeler, D. J. (1998). Understanding industrial experimentation. Knoxville, Tennessee: SPC Press. 


\section{APPENDIX}

March 17, 2017

Mr. Charles Smith

Customer Relations Director

Sony USA

One Sony Drive

Park Ridge, NY 07656

Dear Mr. Smith:

Pictures from a once-in-a-lifetime trip are irreplaceable. I relied on Sony's Cyber-shot DSC-W100 Digital Camera, which I bought for a cruise to the French Caribbean last summer to celebrate my 40th birthday, to record this trip in photographs. Experience, dependability, and customer service are the qualities associated with the name "Sony."

I took hundreds of pictures on my 20-day trip, and although I checked some of the early shots using the LCD screen, I did not check any pictures for the last half of the trip in order to conserve the battery. When I arrived home, however, I discovered that most of the pictures taken later in the trip did not develop because the camera had malfunctioned. Ninety-eight of the pictures taken the last week of the trip were ruined.

As you must realize, the value of these photographs is far greater than the cost of the camera, and replacing the camera is not sufficient. Without pictures, I feel as if I never took the trip; therefore, I am requesting that Sony pay me $\$ 7,500$ so that I may repeat the trip and replace the pictures lost because of this faulty equipment. Enclosed for your examination are the camera, the memory card, the printed photos, and the receipt for the camera.

Sony has an excellent reputation with consumers, and I trust that you will make the correct decision to help me replace my lost memories. Please contact me by April 30 with your response so that I can begin making plans for a fall cruise.

Sincerely yours,

Daniella Davidson

Enclosures: 4 